\title{
Tendencias de lo digital en la arquitectura
}

\author{
Trends in digital architecture
}

$<$ RESUMEN>

La emergencia de lo digital en las diversas disciplinas ha modificado considerablemente las estrategias para capturar, concebir, analizar y comunicar el conocimiento propio de cada una de ellas. De algún modo los expertos afirman que una de las mayores consecuencias de este fenómeno sobre las diversas disciplinas es un incremento en la profesionalización de la práctica de las mismas. Por otra parte, las posibilidades que ofrecen los medios digitales tanto en la velocidad de manejo como en la manipulación de grandes volúmenes de información, redundan habitualmente en la optimización de factores cuantitativos en desmedro de los cualitativos, ignorándose, por tanto, las modificaciones que podrían estar operando al interior de las diversas disciplinas a partir del uso de tecnologías.

Por otro lado, en las disciplinas del conocimiento la concurrencia existe cuando las unidades o etapas de un proceso de producción de conocimiento - generalmente asumidas como secuenciales- se ejecutan en forma paralela o simultánea a partir de mejoras en la sincronización del conocimiento producido y la comunicación de éste mediante un lenguaje compartido. Todo lo anterior es posible gracias a las tecnologías de información que han permitido crear las dinámicas de los procesos de formulación, evaluación y reformulación reemplazando un proceso de diseño lineal aferrado en la evolución morfológica de un objeto determinado.

$<A B S T R A C T>$

The emergence of digital technology in the various disciplines has considerably changed the strategies to capture, design, analyse and communicate the knowledge of each one of these disciplines. To a certain extent, the experts say that one of the major consequences of this phenomenon of the various disciplines is an increase in the professionalization of the practice. Moreover, the potential of digital media, both in the speed of handling and manipulation of large volumes of information often results in the optimization in quantitative factors whilst undermining qualitative ones, ignoring the changes that may be operating inside the various disciplines from the use of technologies.

On the other hand, in the knowledge-based disciplines competition exists when the units or stages of a process of knowledge production <generally assumed as sequential <are executed in parallel or simultaneously from improvements in the synchronization of produced knowledge and its communication through a shared language. All of this is possible thanks to information technologies that have helped create the dynamics of the processes, assessment and reformulation, replacing linear design process which cling to the morphological evolution of a specific object.

<PALABRAS CLAVE >

DISEÑO DIGITAL / COGNICIÓN DEL DISEÑO / DISEÑO BASADO EN CONOCIMIENTO

¿KEY WORDS

DIGITAL DESIGN / DESIGN COGNITION / KNOWLEDGEBASED DESIGN
A lo largo del desarrollo de la década pasada, nos pudimos percatar del impacto que las tecnologías de la información han comenzado a tener sobre casi todos los aspectos de la vida cotidiana. El fácil acceso a más formas de recibir y manipular información, ha influido fuertemente en el desarrollo de las diversas disciplinas profesionales. La arquitectura, el diseño y la geografía han sido fuertemente afectadas por este fenómeno desde hace algunos años y han tenido que cuestionarse en todos sus aspectos. Algunos de éstos se han visto favorecidos por un indiscutible progreso, sobre todo en lo concerniente a los intercambios de información que confluyen en la generación de un proyecto y su posterior realización (Mitchell, 1994, Sanders, 1996, Schmitt, 1999) ${ }^{1}$.

Dr. Eduardo Lyon R. Arquitecto y académico de la Universidad de Chile, Master en Arquitectura con especialización en diseño digital de la Universidad de Maryland y Doctor en Arquitectura (PhD) con especialización en Diseño Computacional del Georgia Institute of Technology.

Todos estos autores revisan las implicancias del uso de tecnologías digitales en arquitectura. En particular Mitchell, hoy a la cabeza del Media Lab en MIT, realizó predicciones acerca de los cambios en la realidad construida y de nuevos escenarios a partir de la ubicuidad de las tecnologías digitales. Es importante destacar que alguna porción de estos escenarios en particular los concernientes a la práctica de la arquitectura son parte de la realidad cotidiana de muchas oficinas de arquitectura en el mundo, ver: Mitchell, W.J., Malcolm, M. Digital Design Media, Van Nostrand Reinhold. Nueva York, 1994; Wilson, R.K., Frank, C. The MIT encyclopedia of the cognitive sciences. Cambridge, MA, 1999. 


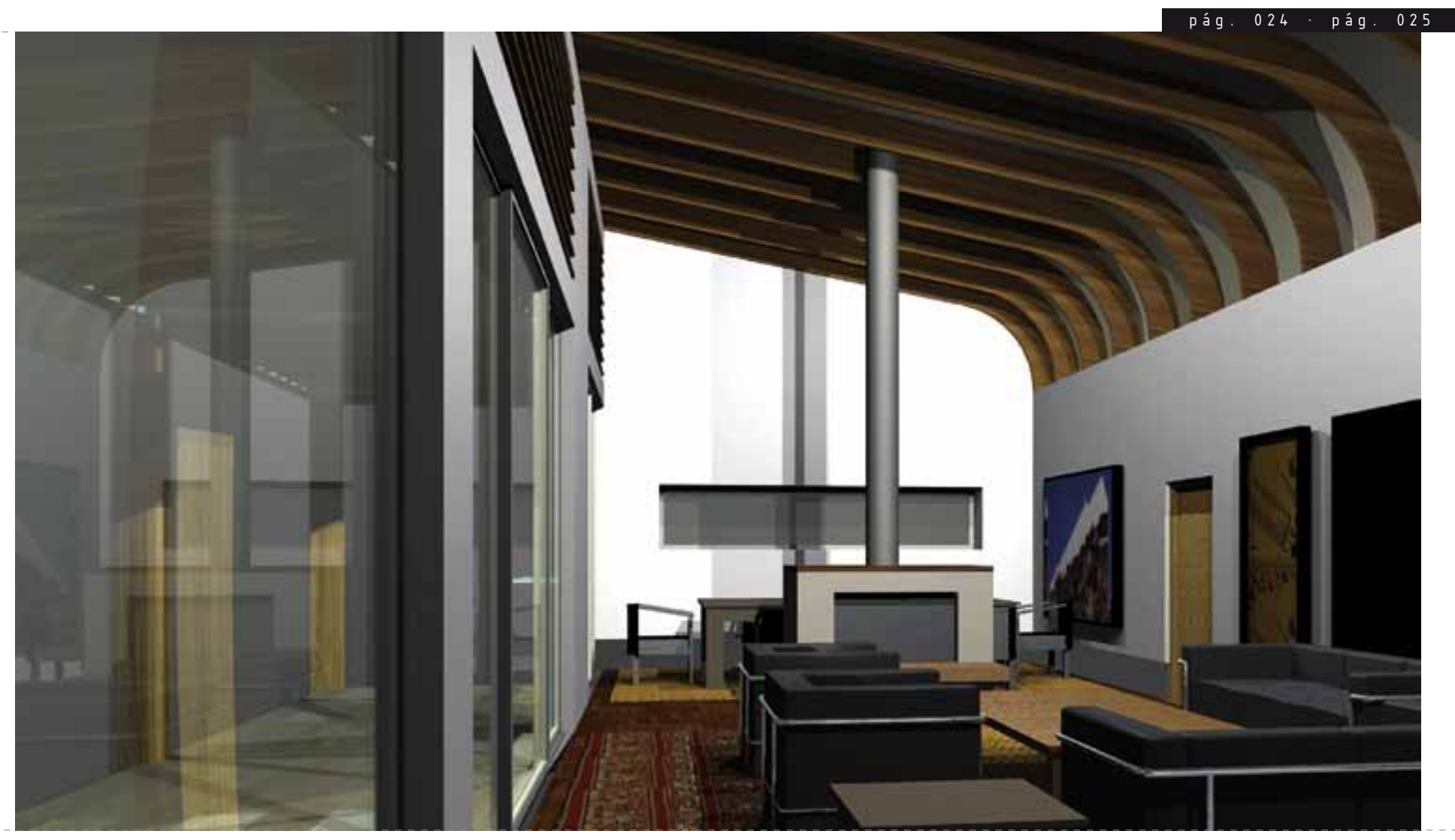

Por otro lado a pesar de que las tecnologías digitales, y en específico los sistemas de diseño asistido por computador (CAD), han sido profusamente introducidos tanto en la educación como en la práctica de la arquitectura, geografía y el diseño, su implementación todavía opera bajo un paradigma propio de los métodos tradicionales², por ejemplo, los sistemas CAD son utilizados como tableros de dibujo digitales ${ }^{3}$. Posteriormente nuevos esquemas de utilización fueron originados e incluidos en las versiones más recientes de los programas $\mathrm{CAD}^{4}$.

Con la ubicuidad del modelamiento tridimensional, nuevos modelos para procesos de diseño han sido desarrollados sobre todo en ambientes académicos ${ }^{5}$. El mismo fenómeno está ocurriendo, también en el nivel académico, producto de la disponibilidad de nuevas técnicas de prototipado rápido y manufactura digital ${ }^{6}$. Por otro lado, los procesos del diseño en la práctica de la arquitectura permanecen inalterados, asociados a las viejas prácticas y a paradigmas obsoletos. Resultado de este modelo, que utiliza los sistemas CAD como herramienta de dibujo bidimensional, y encubiertas bajo el notable aumento en la productividad, a partir del uso de estos sistemas, las reales potencialidades en la utilización creativa de estas tecnologías permanecen ignoradas.

La emergencia es un concepto que describe descubrimientos y/o fenómenos inesperado, o fenómenos globales a partir de la conjunción de comportamientos o condiciones locales. Proveniente del campo de la inteligencia artificial y de la biología, la emergencia describe un fenómeno no determinista, de autoorganización que se hace posible a parti de la interacción local entre las unidades menores dentro de un sistema ${ }^{7}$. Por lo mismo este concepto tiene relación con los conceptos de autoorganización y superveniencia y se define en oposición a los conceptos de reduccionismo y dualismo.

En el campo del diseño la mayoría de las interpretaciones que se hacen de este concepto parecen algo erróneas o simplistas puesto que utilizan interpretaciones limitadas del concepto ${ }^{8}$. La comunidad de investigación

\footnotetext{
Esto fundamentalmente se refiere a la baja adopción del modelamiento tridimensional como instrumento fundamental de diseño, tecnología cual fue introducida formalmente en la carrera de arquitectura de nuestra Universidad, por el profesor Lyon, en el año 1998.

Kostof, S. The architect. Chapters in the history of the profession. 2000.

En una reciente reedición de este libro Dana Cuff realiza un análisis muy certero acerca de las consecuencias de la introducción de los computadores en la educación y práctica de la arquitectura.

Kalay, Y. Architecture's new media: Principles, theories, and methods of computer-aided design. Cambridge MA, Londres, Inglaterra: MIT Press, 2004 Leach, N. Designing for a digital world. Chichester, Inglaterra: Wiley-Academy, 2002.

Kostof, S. Op. cit.

Allen, S. Practice: Architecture, technique and representation. Amsterdam: G+B Arts International, 2000.

Leach, N. Op. cit.

Schodek, D., Bechthold, M., Griggs, J.K., Kao, K., Steinberg, M. Digital design and manufacturing: CAD/CAM Applications in architecture. Hoboken, NJ: Wiley, 2004. Kolaveric, B. Architecture in the digital age: Design and manufacturing. Londres, Inglaterra: Spon Press, 2003.

Kieran, S., Timberlake, J. Refabricating architecture. Nueva York: McGraw-Hill Companies, 2004.

Leach, N., Turnbull, D. Williams, C. Digital tectonics. Chichester, Inglaterra: Wiley-Academy, 2004

Wilson, R.K., Frank, C. The MIT encyclopedia of the cognitive sciences. Cambridge, MA, 1999.

8 Lyon, E., Knoespel, K. «Autopoesis and emergence in architecture». En: Abrioux, I. Conversations: Enacting new synergies in arts and sciences. París, Francia: Society for Literature and Science, 2004

Poon, J., Maher, M.L. «Co-evolution and emergence in design». Artificial Intelligence in Engineering 1997; 11: pp. 319-327.
} 


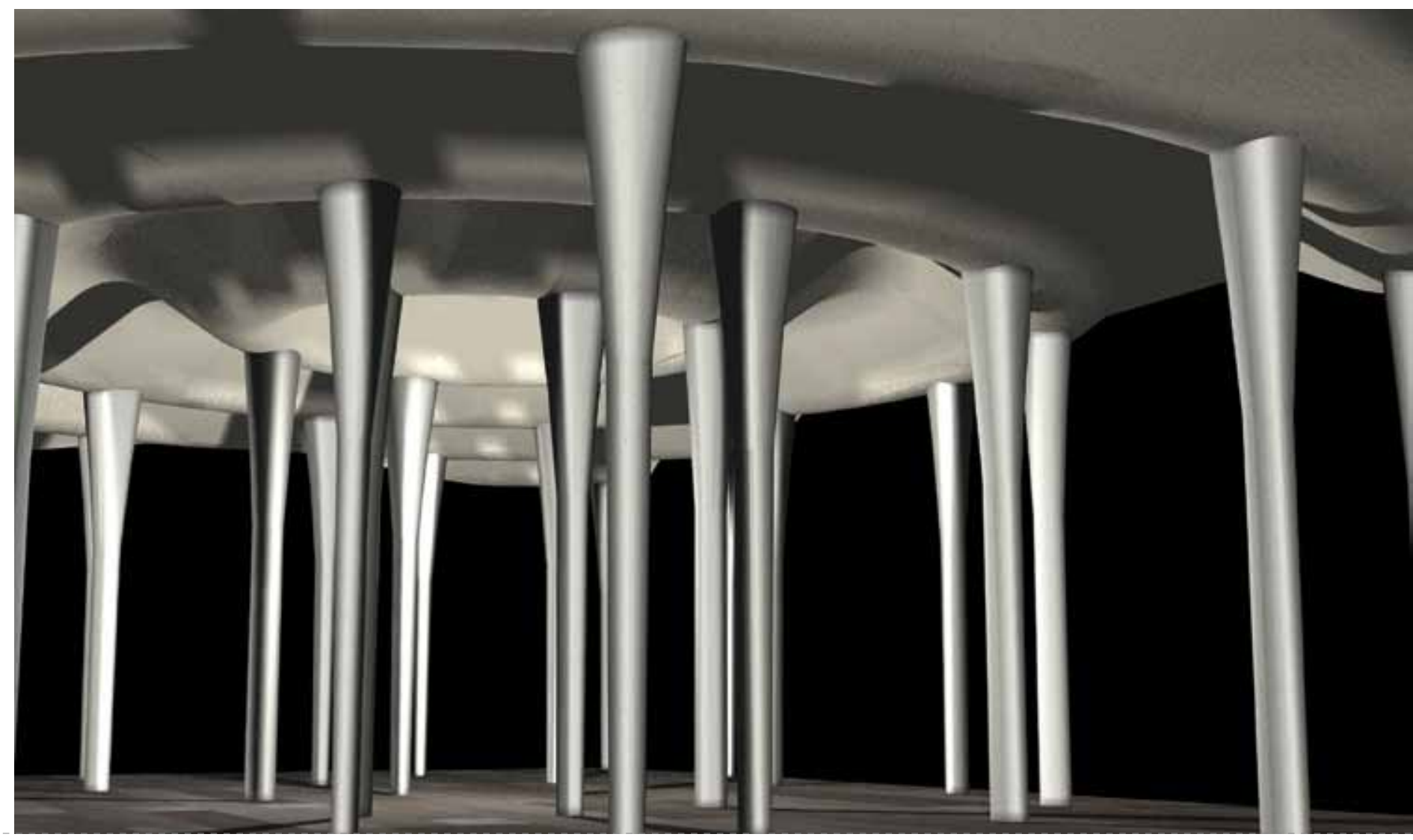

del diseño que trabaja con la gramática de diseño o arquitecturas algorítmicas proclama la emergencia como uno de los resultados más relevantes en el uso de estas técnicas de programación y búsqueda9 ${ }^{9}$. Pese al éxito relativo en el uso de estas técnicas resultan evidentes las limitaciones que presentan los modelos lineales de generación de la forma arquitectónica ${ }^{10}$.

En contraste con los modelos tradicionales definidos fundamentalmente por la manipulación abstracta de objetos, este estudio reconoce que los recursos disponibles para repensar nuestra disciplina deben ser encontrados en una reformulación de su teoría en relación a sus procesos de diseño. Esta reformulación se debería basar en una conceptualización de los procesos de diseños entendiéndolos como no lineales y cognitivamente distribuidos. Basados en este paradigma, las dinámicas de los procesos de formulación, evaluación y reformulación reemplazan un proceso de diseño lineal aferrado a la evolución morfológica de un objeto determinado. Por otro lado los avances en computación en la disciplina de la arquitectura, en relación a su formulación teórica y desarrollo, han estimulado la implementación de un número creciente de sistemas de diseño asistido por computador (CAD), análisis asistido por computador (CAE) y manufactura asistida por computador (CAM). La elaboración de estos sistemas responde y ha generado nuevas conceptualizaciones ${ }^{11} \mathrm{del}$ conocimiento de nuestra disciplina ${ }^{12}$.
A modo de ejemplo tenemos que los extensos repertorios de generación formal y las enormes capacidades de manipulación de ellas presentes en los sistemas CAD, están poniendo a disposición de los arquitectos formas de gran valor creativo y con geometrías de alta complejidad ${ }^{13}$. Muchas de las formas producidas a partir de la posibilidad y capacidad de manipulación antes mencionadas, no están relacionadas con sistemas de construcción tradicionales. Las superficies curvas se constituyen como un caso específico en este problema. A pesar de que las superficies curvas han sido parte del vocabulario formal de los arquitectos, su uso ha estado siempre limitado por la falta de instrumentos adecuados para manipular y construir formas que utilizan estas geometrías.

9 Basil, S., Edmonds, E. «The cognitive basis of emergence: Implications for design support». Design Studies 1996; 17: pp. 451-463.

10 Lyon, E. «Architecture and autopoiesis: Notes on design theory, methods, and instruments». International Journal of Architectural Computing $2005 ; 3$ : pp. $317-333$.

1 Cabe destacar que la Facultad de Arquitectura y Urbanismo de la Universidad de Chile ha sido pionera en la implementación de este tipo de sistemas.

2 Leach, N. Designing for a digital world. Chichester, Inglaterra: Wiley-Academy, 2002.

Schodek, D., Bechthold, M., Griggs, J.K., Kao, K., Steinberg, M. Op. cit.

Winograd, T., Flores, F. Understanding computers and cognition: A new foundation for design. Addison-Wesley, 1987.

De Luca, F., Nardini, M. Behind the scenes: Avant-garde techniques of contemporary design. Basel, Boston: Birkhäuser, 2002.

Gehry, F.O. Architecture of Frank Gehry. 1986.

Allen, S. Op. cit.

Pawley, M., Foster, N. Norman Foster: A global architecture. Nueva York: Universe Distributed to the U.S. trade by St. Martin's Press, 1999. 

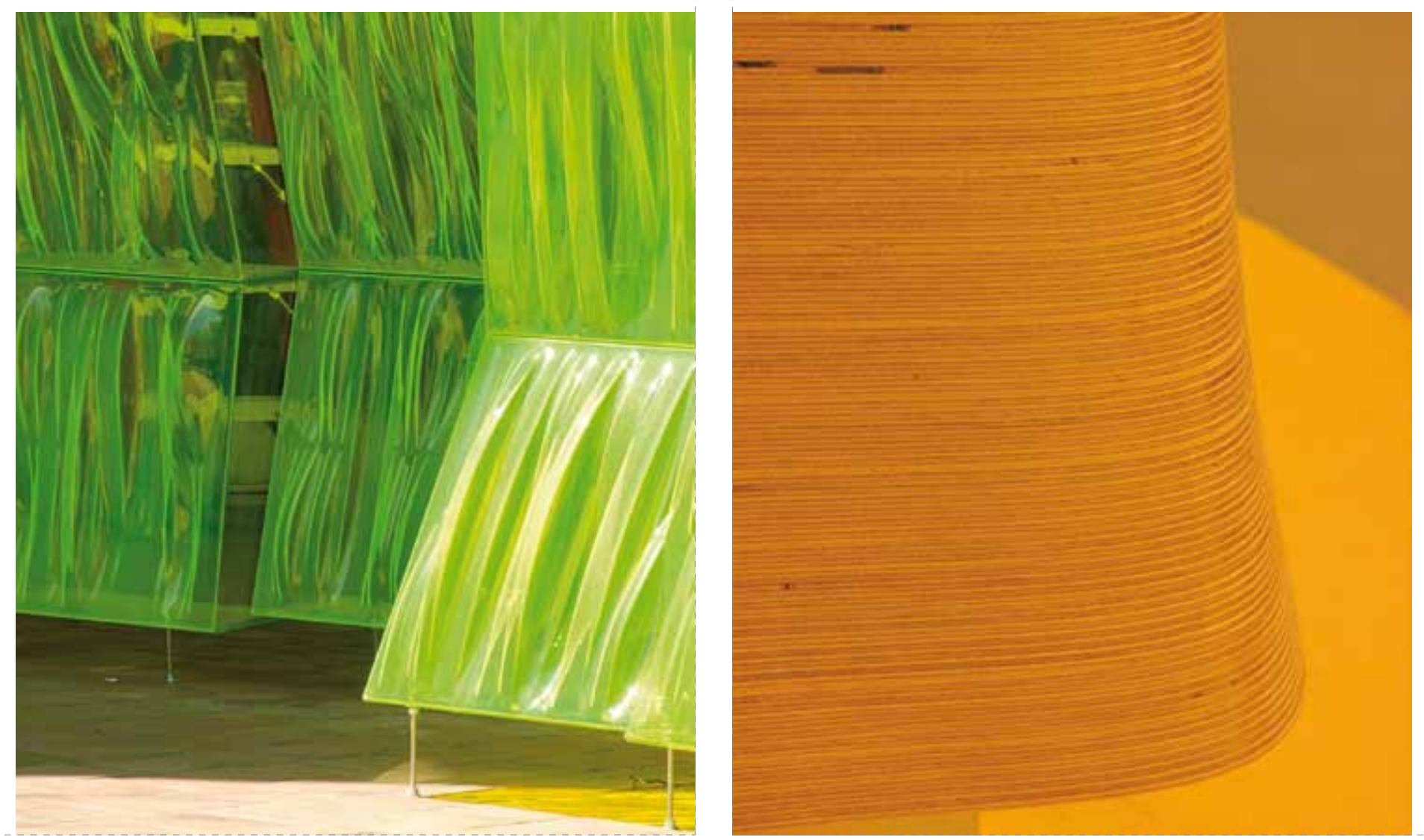

Conforme a lo anterior, y debido al desarrollo y uso extensivo de los sistemas CAD, es posible percibir un notable incremento en el uso de geometrías complejas en general y superficies curvas en arquitectura. Los métodos de construcción para poder fabricar estas formas no son estables ni determinados y generalmente incluyen el uso de tecnologías de manufactura digital incluyendo sistemas de manufactura asistida por computador (CAM) y maquinaria controlada por computador (CNC). El uso de maquinaria CNC en la producción de edificios nos está dando una idea de cómo la producción de arquitectura evolucionará en el futuro ${ }^{14}$. De algún modo, las tecnologías de diseño y manufactura digital constituyen un alejamiento definitivo de los modelos de construcción liderados por un contratista y basados en el trabajo en el lugar de la obra. Consecuentemente la organización de la producción de edificios no estará limitada exclusivamente a los sistemas tradicionales de construcción ${ }^{15}$. La manufactura digital representa un cambio sustancial de los modelos de producción en masa en donde la repetición era la base de la economía. A partir de la utilización de sistemas CAM las variaciones y los componentes a pedido no representan un costo adicional por la utilización de mano de obra especializada o técnicas de manufactura especial16.

El diseño puede ser pensado como la composición y refinamiento de ciertos conceptos y tecnologías, mientras que la construcción se podría pensar como la descomposición del diseño en sus partes producibles y sus correspondientes procesos de ejecución. Mediante el uso de tecnologías digitales las unidades de diseño son posibles de analizar y descomponer para su construcción, de este modo el espacio de las posibles alternativas y de los potenciales errores de construcción se reduce exponencialmente, como también los riesgos de cambios durante el proceso de construcción ${ }^{17}$.

Esto se vuelve especialmente relevante si nos damos cuenta que la proporción de problemas de baja productividad y mala calidad de la construcción se mantiene cerca del treinta por ciento. Del porcentaje anterior cerca del cincuenta por ciento es atribuible

14 Callicott, N. Computer-Aided manufacture in architecture - The pursuit of novelty. Elsevier Science \& Technology, 2000.

15 Groák, S. The idea of building: Thought and action in the design and production of buildings. Londres, Nueva York: E \& FN Spon, 1992.

Groák discute ampliamente el asunto del conocimiento de construcción en la producción de edificios en el capítulo once Transferencia tecnológica y en específico la literatura relacionada en la página 167. Adicionalmente el se extiende acerca de las diferentes formas de conocimiento de edificación en las páginas 169-170.

16 Schodek, D., Bechthold, M., Griggs, J.K., Kao, K, Steinberg, M. Op. cit. Kolaveric, B. Op. cit.

Stacey, M. Component design. Oxford, Boston, MA: Elsevier Science \& Technology, 2001.

17 Lyon, E. Component Based Design and Digital Manufacturing: A DfM Model for Curved Surfaces Fabrication using Three Axis CNC Router. eCAADe 2006 - Digital design: Communicating Space(s)eC AADe 2006, septiembre 6-9 Lisboa, Volos, Grecia. eCAADe (Education and Research in Computer Aided Architectural Design in Europe). 2006 
a errores de diseño ${ }^{18}$. En la concepción tradicional del diseño arquitectónico, un edificio es considerado como un sistema compuesto de subsistemas (estructural, espacial y funcional), y la principal labor de los arquitectos consiste en determinar la ubicación espacial, interacción y coordinación de estos sistemas guiados por una gramática formal estilo o estética dominante, mediante el uso de dibujos bidimensionales. Tradicionalmente la construcción se basa en el moldeado a partir de materiales en bruto y en procesos de ensamblaje de componentes en terreno. Ahora en las últimas décadas hemos visto un cambio progresivo a un modelo basado en el armado y ensamblaje en terreno de partes y componentes manufacturados fuera del sitio de construcción, esto es lo que se denomina el modelo de ensamblaje de componentes ${ }^{19}$.

Tanto en la industria manufacturera como en la industria de la construcción es ampliamente reconocido que la productividad y la calidad pueden ser incrementadas a partir de la integración del conocimiento acerca de la producción en las etapas de diseño.

Al interior de la industria manufacturera diferentes formas de integración del conocimiento de la producción ha conducido a mejoras sustanciales en la productividad y la calidad. Sin embargo en el ámbito de la industria de la construcción se caracteriza por su constante baja en la productividad y empobrecimiento en la calidad de los edificios ${ }^{20}$. El proceso tradicional de documentación de un proyecto de Arquitectura produce descripciones detalladas de los sistemas y subsistemas que componen un edificio, donde la interacción entre estos sistemas y subsistemas es asumida como parte del trabajo de coordinación del arquitecto. La fabricación de componentes y los diferentes procesos de ensamblaje de éstos permanece implícita hasta el Ilamado a propuesta e incluso hasta el inicio de la ejecución de la obra.

El problema fundamental es que aún se ignora el hecho que hasta el setenta por ciento de los costos de producción es determinado en las etapas de diseño ${ }^{21}$, los arquitectos están preocupados mayormente en describir y localizar sistemas y subsistemas en el espacio usando dibujos bidimensionales, mientras que los contratistas o constructores se preocupan de la producción de componentes y partes, y de la implementación de procesos de ensamblaje en el tiempo a partir de estos dibujos. Obviamente es necesaria una mejor integración y coordinación a partir de mejores representaciones, que sean más eficaces y eficientes.

La concurrencia es la simultaneidad de ocurrencia de hechos o sucesos dentro de un sistema. En arquitectura la concurrencia existe cuando las unidades o etapas de un proyecto -generalmente asumidas como secuenciales- se ejecutan en forma paralela o simultánea a partir de mejoras en la sincronización del conocimiento producido y la comunicación de éste mediante un lenguaje compartido. O sea, la idea fundamental es que esta integración se produzca a partir de la coordinación y concurrencia temprana de todos los especialistas que toman parte en el proceso y no, uno después de otro. Todo lo anterior es posible gracias a las tecnologías de información que han permitido crear modelos tridimensionales paramétricos, con bases de datos asociadas, a las que todos los especialistas pueden ir aportando su conocimiento desde el inicio de la gestión del edificio; esto es lo que se denomina el modelo BIM (Building Information Model) o modelo de edificio de datos ${ }^{22}$.

Este concepto plantea cambios fundamentales en el futuro de la industria de arquitectura y construcción. El modelo de edificio virtual plantea la creación de un entorno de trabajo centrado en un modelo tridimensional paramétrico al cual se le integran todas las capas de información proporcionadas por los profesionales participantes desde consultores a usuarios (Eastman, 1999). Este modelo se transforma en el cerebro del edificio no sólo durante la etapa de proyecto y construcción sino que también a posteriori en el uso del edificio. Este modelo se fundamenta en dos conceptos esenciales: el primero es la existencia de un modelo tridimensional del edificio que funciona a la vez como una base de datos en donde se aloja toda la información concerniente al diseño, construcción y uso del edificio. El segundo concepto es la existencia de un lenguaje en este caso XML o IFC (Industry Foundation Classes) ${ }^{23}$ que permite la transferencia y extracción de datos desde el modelo centralizado por parte de todos los integrantes de los equipos profesionales involucrados, en tiempo real. Esta iniciativa permite la integración a partir de modelos 3D paramétricos de modelos estructurales, de instalaciones, detalles de construcción, programación de obras, simulación de

18 Esta información es referenciada por Stephen Fox y proviene de dos orígenes: Barber, P., Graves, A., Hall, M., Sheath, D., Tomkins C. «Quality failure costs». Internationa Journal of Quality \& Reliability Management 2000; 17(4): pp. 479-92; BRE. Quality control on building sites, building research establishment current paper $7 / 81$, HMSO. London, 1981.

19 El modelo de ensamblaje-componente es denominado por Fox como el modelo «liderado por el productor». De manera similar otros autores como Groák usan el término modelo «liderado por el manufacturero». Todos ellos refieren a la producción de partes y componentes en fábricas y talleres fuera del sitio de construcción de acuerdo a especificaciones de diseño y posteriormente ensamblados en terreno. Fox analiza en profundidad lo que Steven Groák enuncia en Groák, S. The idea of building: Thought and action in the design and production of buildings. Londres, Nueva York: E \& FN Spon, 1992; p. 174. Groák realiza un análisis en profundidad del proceso de edificación en el capítulo siete, páginas 121-130. Adicionalmente. Groák profundiza en los aspectos relativos a como se organiza el conocimiento en la producción de edificios, ver capítulo ocho, páginas 132-133.

20 En relación con el incremento de la productividad y la calidad de la construcción mediante el diseño en sector manufacturero véase: Peck, H. Designing for manufacture. Londres: Pitman, 1973. En la misma temática pero relacionada con el sector de la construcción véase: Emmerson, H. Survey of problems before the construction industry. Londres: Ministry of Works, 1962. En relación a los avances en la industria manufacturera véase: Dean, J.W., Susman, G.I. «Organizing for manufacturable design». Harvard Business Review 1989; 28-36. Para una revisión de la caída en la productividad en la industria de la construcción en el Reino Unido véase: DETR. Rethinking construction. The report of the construction industry task force. Londres: Department of the Environment. Transport and the Regions, 1998. Para una mejor revisión de la caída en la productividad de la industria de la construcción en Estados Unidos véase: Allmon, E., Haas, C.T. Borcherding J.D. Goodrum, P.M. "U.S. construction labor productivity trends, 1970-1998". Journal Of Construction Engineering And Management 2000; 97

21 Giachetti, R.E. «A decision support system for material and manufacturing process selection». Journal of Intelligent Manufacturing 1998; 9: pp. 265-276. Boothroyd, G., Dewhurst, P., Knight, W.A. Product design for manufacture and assembly. Nueva York: Marcel Dekker, 2002. Giachetti hace referencia a: Ullman, D.G. The mechanical design process. Nueva York: McGraw-Hill and Boothroyd References Munro and Associates Inc, 1992.

22 Finalmente cabe destacar que el profesor Lyon es experto en el modelamiento de edificios usando el modelo de edificio de adtos o BIM (Building Information Model). El creador de esta tecnología es Charles Eastman director del programa de doctorado en el Georgia Institute of Technology, que a la vez fue el profesor guía del profesor Lyon en su investigación Doctoral.

23 IFC consiste en el estándar producido por la Alianza Internacional para Interoperatividad (IAI) con el fin de facilitar la transferencia e extracción de datos desde el modelo centralizado por parte de todos los integrantes de los equipos profesionales involucrados en la producción, uso y reciclaje de un edificio. 


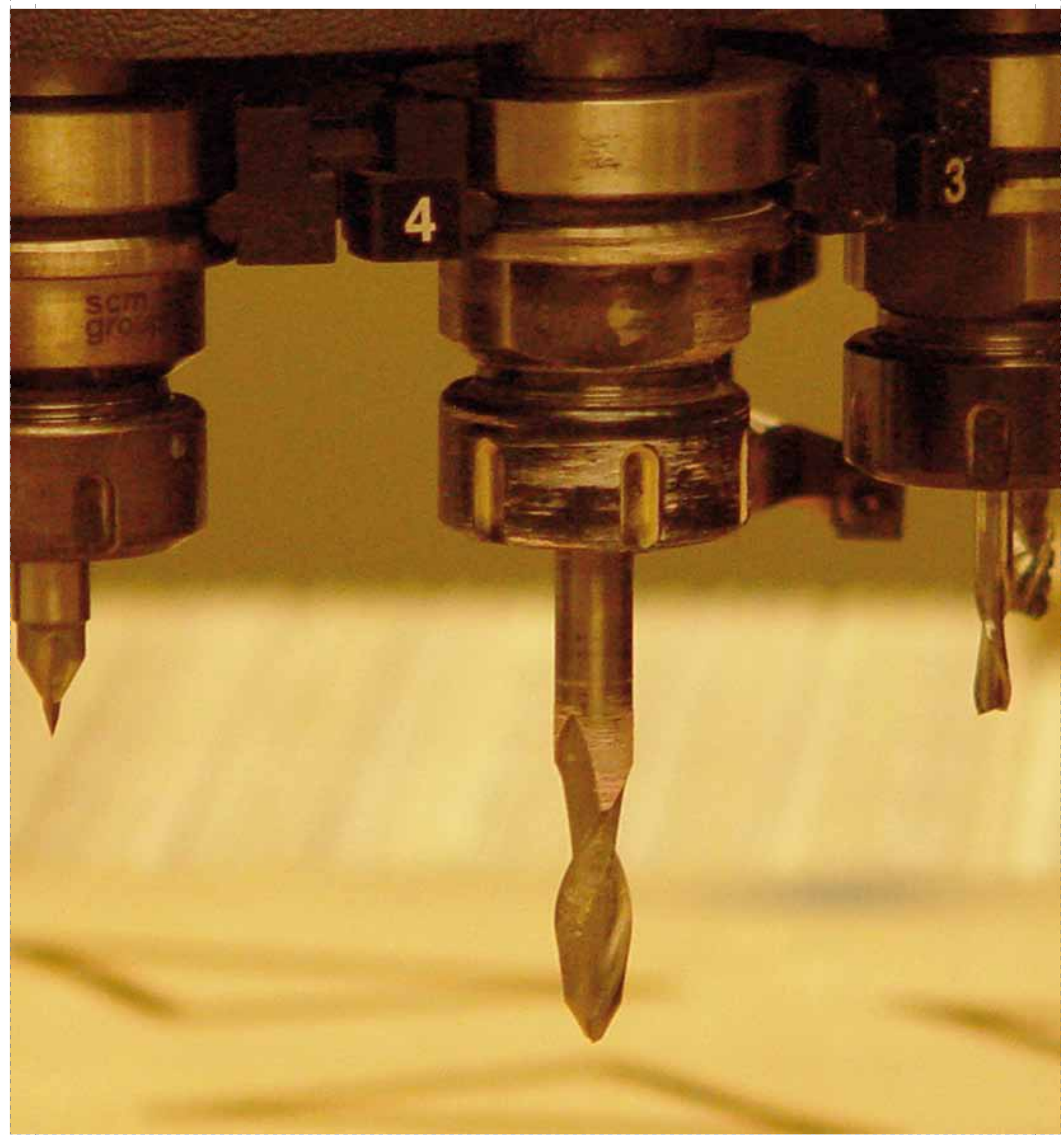




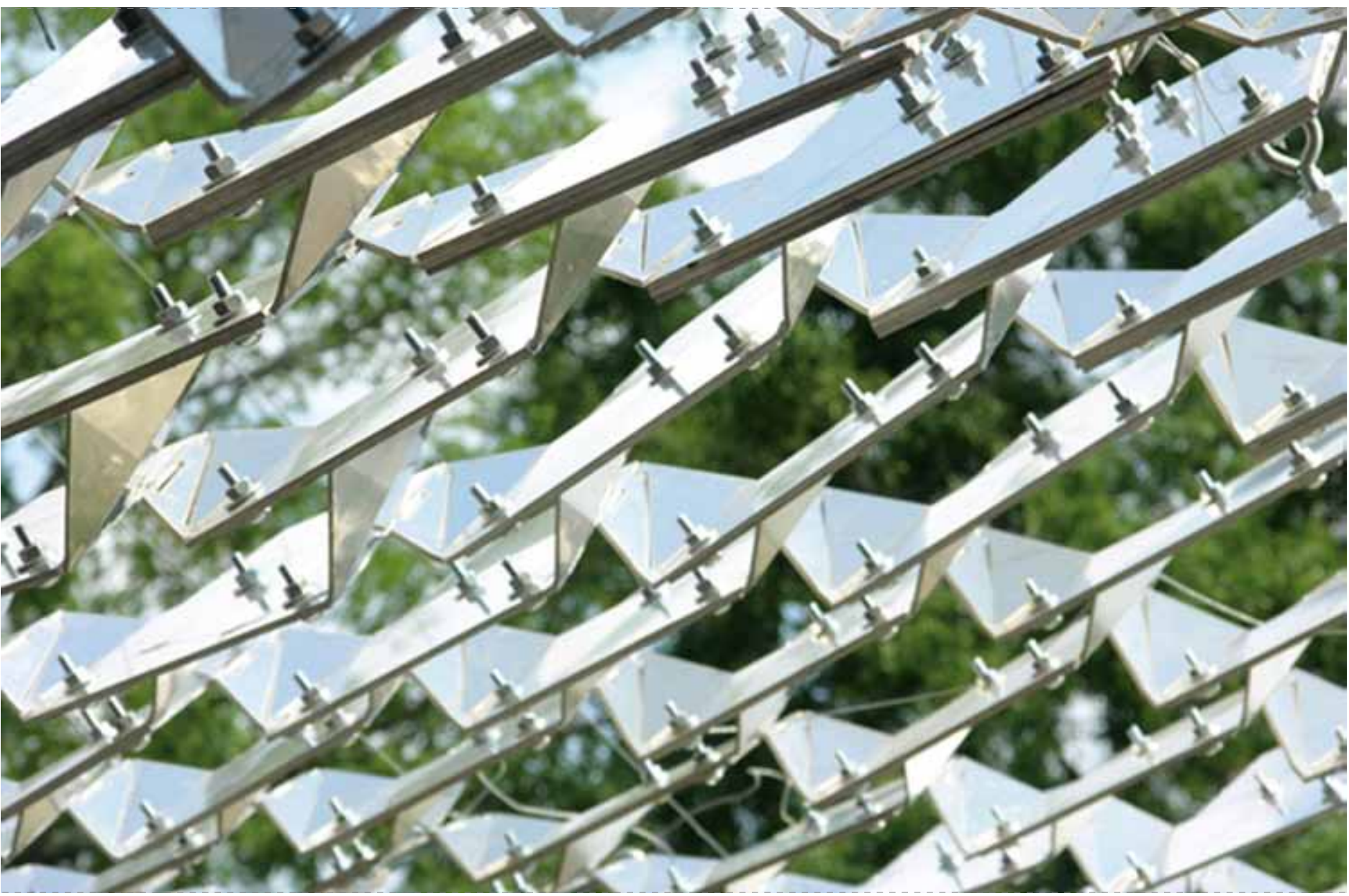

procesos de construcción o 4D, sitios web que proveen servicios, materiales y componentes para los edificios ${ }^{24}$

La llegada de las tecnologías CAD, CAE y CAM y su implementación a partir del modelo BIM en la producción de edificios han introducido un entorno de trabajo totalmente distinto al tradicional, que acarrea cambios undamentales al interior de las disciplinas relacionadas con la producción, uso, administración y reciclaje de edificios. Este nuevo entorno de trabajo se constituye a partir de un cambio en la tradicional separación entre las intenciones de diseño, los medios de ejecución y las modalidades de uso de un edificio. A la vez deja a la vista no solamente las ineficiencias derivadas de la división entre diseño, análisis, y construcción de un edificio (Moore, 1996) sino que también de la predominancia de los modelos secuenciales en el diseño arquitectónico (Lyon and Knoespel, 2004).
Fundamentalmente estas especiales circunstancias ofrecen la oportunidad a los arquitectos de liderar esta nueva forma de producción de edificios, recobrando de algún modo el rol renacentista de «maestro de obras» y reestableciendo el vínculo entre arquitectura y construcción a partir de procesos de diseño basados en la captura, generación y manipulación de conocimiento a partir de la utilización creativa de las tecnologías digitales ${ }^{25}$.

24 El modelo BIM es considerado como eje fundamental en la producción de edificios en el futuro. Reafirmando lo anterior y en base a la creciente evidencia del significativo aumento en la productividad a partir de su implementación el gobierno americano exige su uso desde el año 2006 en cualquier edificio de fines gubernamentales.

25 Kieran, S., Timberlake, J. Refabricating architecture Nueva York: McGraw-Hill Companies, 2004.

Kolaveric, B. Architecture in the digital age: Design and manufacturing. Londres, Inglaterra: Spon Press, 2003.

Moore, D. «The renaissance: The beginning of the end for implicit buildability». Building Research and Information 1996; 24: pp. $259-269$. 